\title{
Smart solution for reducing COVID-19 risk using internet of things
}

\author{
Akshaya Rajeshkumar, Senthilkumar Mathi \\ Department of Computer Science and Engineering, Amrita School of Engineering, Coimbatore, Amrita Vishwa Vidyapeetham, India
}

\begin{tabular}{|c|c|}
\hline Article Info & ABSTRACT \\
\hline Article history: & The article exposes a smart device designed for mitigating the coronavirus \\
\hline Received May 6, 2021 & disease (COVID-19) risk using the internet of things. A portable smart \\
\hline Revised Nov 10, 2021 & alert people when the guidelines given by the government were not followed \\
\hline Accepted Nov 23, 2021 & $\begin{array}{l}\text { and alert health authorities when any abnormalities found. By doing so, the } \\
\text { spread of this fatal disease can be stopped. The modules of the proposed }\end{array}$ \\
\hline Keywords: & module, crowd detection and analysis module, health screening module and \\
\hline Face recognition & health assessment module. The proposed system can be placed in any public \\
\hline Health screening & $\begin{array}{l}\text { entrances to monitor people without human intervention. Firstly, the human } \\
\text { face images are captured for face mask check, then the crowd analysis of the }\end{array}$ \\
\hline Portable smart alerting device & $\begin{array}{l}\text { particular entrance where the person is entering is performed, thereafter } \\
\text { health screening of the person is done and the values were imported to the } \\
\text { health assessment module to check for any abnormalities. Finally, after all } \\
\text { the conditions were met the door is opened automatically. The smart device } \\
\text { can be installed and effectively used in many scenarios such as malls, stores, } \\
\text { crowded places and campuses to avoid the risk of spread of the coronavirus. }\end{array}$ \\
\hline
\end{tabular}

This is an open access article under the CC BY-SA license.

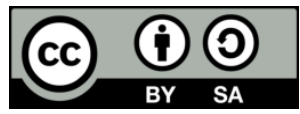

\section{Corresponding Author:}

Senthilkumar Mathi

Department of Computer science and Engineering, Amrita School of Engineering

Coimbatore, Amrita Vishwa Vidyapeetham, India

Email: m_senthil@cb.amrita.edu

\section{INTRODUCTION}

The global calamity coronavirus disease (COVID-19) tends to be immensely troublesome for mankind due to its adverse effects. The entire world was under lockdown to battle this pandemic. Many types of researches are carried out for the invention of vaccine to bring everything back to normal. Since this disease is not mitigating without a vaccine, it is really necessary to make efficient use of advanced technologies [1]. This pandemic is the hardest battle to overcome as it has adverse health effects which finally leads to death.

Strict laws on social distancing, lockdown, wearing face masks, using sanitizers and regular health checkups were imposed. The entire universe was feeling distressed and terrified due to the spread of this pathetic disease. The pandemic caused huge damage to the manufacturing sector. The pandemic leads to mass unemployment, failures in economic activities and world trade experienced one of the worst downfalls [2]. It spread rapidly to almost all the countries across the globe and the death rates were increasing day by day. The coronavirus affected people in different ways but the most common symptoms are fever, dry cough and tiredness. The coronavirus can also spread when a person is in contact with an infected person. Because the virus can spread easily and rapidly when one is near the infected person as the person tend to breathe the 
infected air or touch the contaminated surfaces. To avoid the spread of this disease, one must maintain social distance, wear face masks properly without fail and wash hands with sanitizers regularly [3].

Once any abnormalities discovered the person must immediately report to the hospital to avoid further spread and to get it cured at the earliest. The government has given several guidelines to safeguard the public and the entire nation. Following those guidelines and being aware can stop the spread any further. It is found that the coronavirus firstly affects the respiratory system severely and then cause damage to other organs thereby ending up in death [4]. Therefore, an immediate remedy to avoid this fatal disease must be found [5]. Various studies on producing the vaccines are carried out by many researchers all over the world but the use of advanced technologies in a smart way can help to mitigate this widespread.

Internet of things (IoT) and internet protocol mobility is considered to be one of the vital technologies [6], [7]. It reduces human efforts and make the devices smart enough to perform the tasks done by human beings effectively to produce the best solutions. Many smart devices and methods were proposed which helped people to maintain social distancing and also to follow other precautions given by the government [8], [9]. Many cost-effective robots, drones and smart devices were designed to help deliver the necessary food items and other essentials to people at the doorstep. This was found to be very useful during the lockdown [10]. Some smart devices helped to detect the symptoms of the disease within seconds [11]. Using the advanced technologies individuals were able to get their treatment done online and quarantine themselves at home. Hence, the proposed novel smart system can help the individuals and public to be more cautious by obeying all the laws and therefore stop the spread of the coronavirus.

\section{RELATED WORK}

There are many useful smart devices and solutions proposed by various researchers to reduce the risk of the spread of the disease. The paper discusses a smart device designed for alerting people when the social distance was not maintained [8]. Along with this feature, this device had an additional feature to indicate the symptoms of the pathetic COVID-19 disease. The work in [10] proposed an approach where face-to-face interactions and physical contacts were avoided by using smart city technology. Drones and robots were used to supply the necessary services to people. A study was made on the need for exploring and indicating the best IoT applications which provided a solution for battling the COVID-19 disease. An outlook on all the smart applications and approaches were explored in the work along with their drawbacks [1].

Yan et al. [5], the work elaborates on the major areas of research that were highly impacted by the pandemic. The paper aims to identify the root cause of this disease and explore more on the research areas related to it. It mainly creates awareness among people and aims at educating them on the importance of being cautious and following the guidelines given by the government. Ten major technologies which helped to lessen the risks of COVID-19 was discussed [12]. Daily updates of infected people were provided accurately with the use of surveillance systems. The author has emphasized the benefit of advanced technologies which helped in ensuring public health. Many other creative ideas and approaches for tackling the worst scenario caused by the pandemic were also discussed.

A framework for accurate face detection using the integral image was discussed in [13]. The critical visual features were extracted using the Adaboost algorithm and the desired portion alone was obtained very quickly. The unwanted background regions were removed to make the computation faster. This system provided the best results compared to any other previous methods. Face detection using the Microsoft HoloLens was discussed in [14]. Detection rates were twelve percent higher than the existing Viola-Jones algorithm. More accurate detections were made within seconds. A smart medical device intended for indicating the symptoms of COVID-19 was designed. It made use of headsets and mobiles for indicating the symptoms of the disease. This smart device was of low cost and had a flexible design [15].

\section{PROPOSED SYSTEM}

The proposed system consists of four main modules as shown in Figure 1. It can be placed in any public entrances to make sure whether the people are aware and are following the guidelines given by the government. The main advantage of this smart device is that it can monitor people without human intervention and with higher accuracy.

The first module is the face mask detection module which was able to detect the face masks using the Viola-Jones algorithm. Then the crowd detection module performed crowd analysis of the particular place where the smart system was deployed [16]. There was an alert when the place was crowded so that the person was not allowed to enter inside. The health screening module performed a health check by measuring the temperature and heart rate of the person [17]. Then, the health assessment module produced the screening results accurately and alerted the health authorities when abnormalities were found. When there were no 
abnormalities, hand sanitization was done using the non-contact portable sanitizer. Finally, after all the conditions were met the door was opened automatically. This system is novel and produced results with higher accuracy [18]. This smart device would be the best solution to remind people to be aware and follow all the precautions to stop the spread of the COVID-19 disease [19].

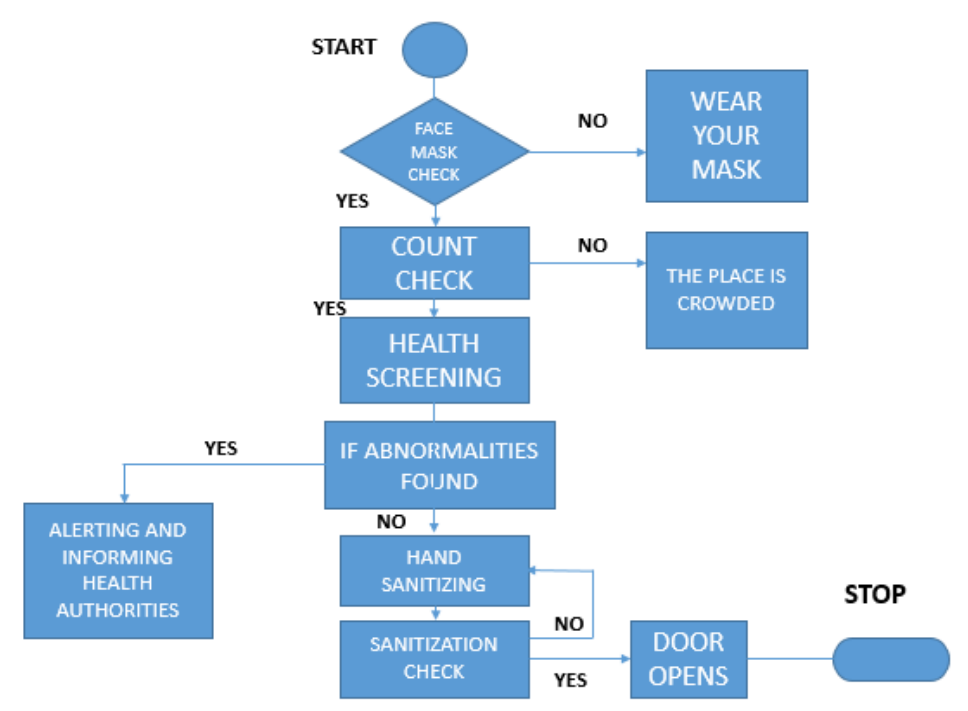

Figure 1. Weibull distribution of all filler concentrations

\subsection{Methodology}

Face mask check and crowd analysis were performed using one of the best real-time face detection (Viola-Jones algorithm). It was able to produce accurate results very fast in real-time. Figure 2 depicts the block diagram of the proposed system. The hardware used in the proposed system were ESP32 microcontroller, infrared sensor, alcohol sensor, relay, valve, L293D motor driver circuit, direct current (DC) motor, ultrasonic sensor and oximeter as shown in Figure 2. The software requirements were MATLAB, Arduino IDE and Embedded C. The data was stored in the cloud. MATLAB software was used for face recognition and crowd analysis. It detected whether the person was wearing a mask or not to alert the person when the mask was not worn.

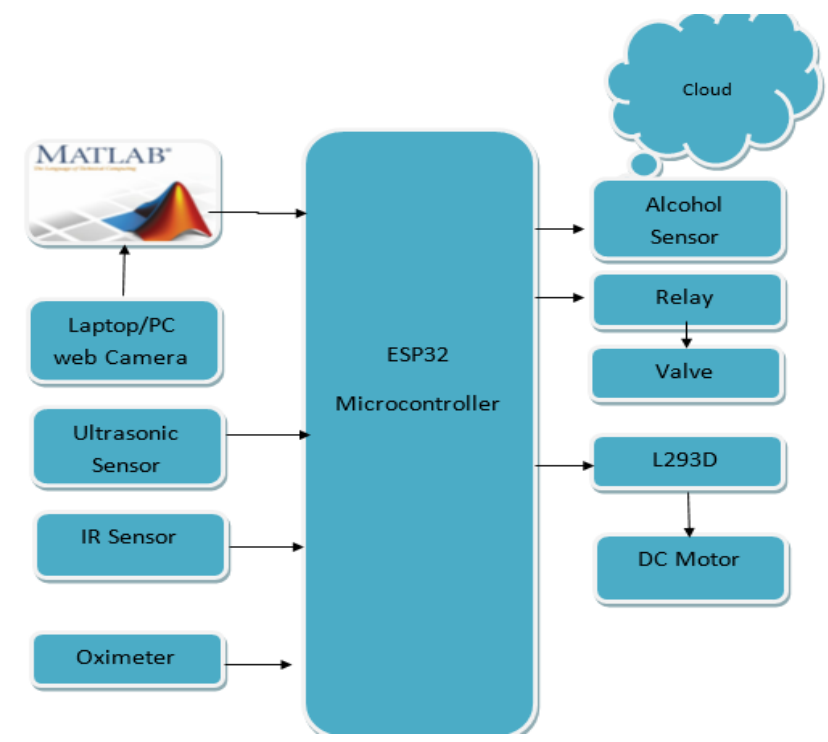

Figure 2. Block diagram of the proposed system 
An ultrasonic sensor helped to monitor social distancing. It sent an alert to the person immediately when some other person was near him/her in the range of six feet. The infrared sensor was used for measuring the body temperature and an oximeter for measuring the oxygen saturation level in the blood [20]. For automatic sanitization infrared sensor was used to sense the presence of the human and by using a relay and valve, the sanitization was done. After the sanitization process, the alcohol sensor detected whether the person was sanitized or not. When all the conditions were met, the door automatically opened. The data was updated in the Google Firebase cloud which was used for data monitoring.

\subsection{Algorithm}

The Viola-Jones algorithm was implemented in the proposed system. It proves to be one of the efficient algorithms for providing accurate results at a faster rate in real-time [21]. For enhancing the focus, this algorithm has been utilized by the camera and smartphone manufacturers. The algorithm has some excellent features like Haar features and Adaboost features which helps for faster face detection [14]. ViolaJones algorithm proves to be one of the best and simplest approaches to provide accurate results for face detection in real-time. The algorithm has exceptional methodologies and features which helps for faster face detection.

Firstly, the input must be fed as shown in Figure 3. Haar-like features are then identified. Features are classified using the integral image. Accurate extraction of the exact features is done using the integral image [22]. To remove the unnecessary background Adaboost learning method is used. It can also generate the desired portion in the face image. Hence, it contributes to easier computation and the cascaded classifier is the efficient face candidate selector. It can accurately identify a face or faces from any given image [23]. Therefore, this algorithm efficiently detects the face masks by the above mentioned four key concepts. The images obtained for face recognition can be secured further by adding face authentication algorithms and other image processing techniques [24]. The results are communicated to the user, healthcare authorities, and alerting them [25].

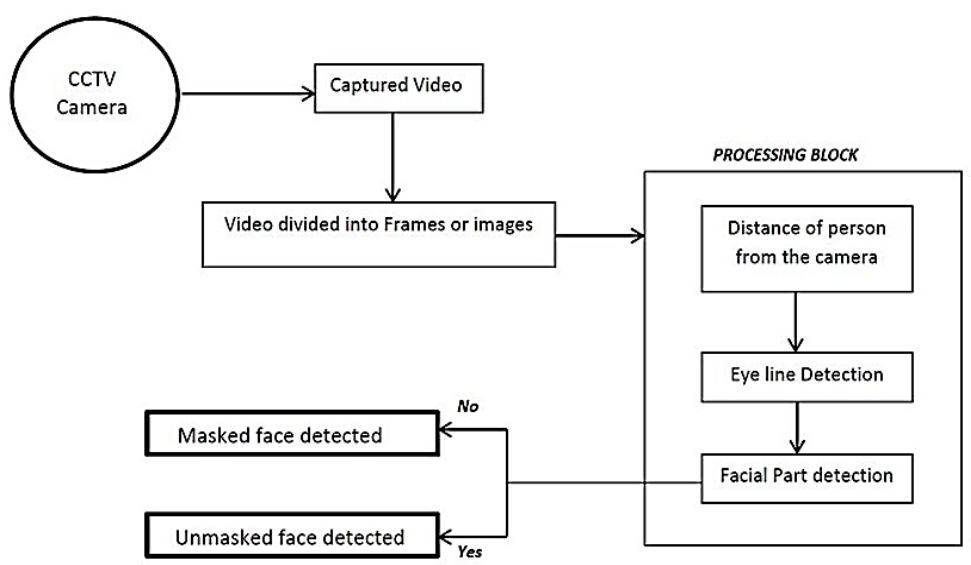

Figure 3. Face mask detection

\section{RESULTS AND DISCUSSION}

Face mask detection along with crowd detection was done in the MATLAB software by implementing the Viola-Jones algorithm. The hardware and software were serially connected using the universal serial bus (USB) cable. The facemask detection results were obtained with higher accuracy as shown in Figure 4. It was capable of figuring out even the slight differences when the masks were not worn properly. The hardware setup was made and the health screening results were obtained and assessed with accuracy. The proposed system was capable of detecting the face masks with an accuracy of 97.76\%. The algorithm worked well for different categories as well. The accuracy for each category is shown in Table 1.

Table 1. Accuracy for different categories

\begin{tabular}{lc}
\hline \multicolumn{1}{c}{ Category } & Precision in percent \\
\hline Front facing & 98 \\
Facing left & 91 \\
Facing right & 89 \\
Looking up & 81 \\
Looking down & 80 \\
\hline
\end{tabular}




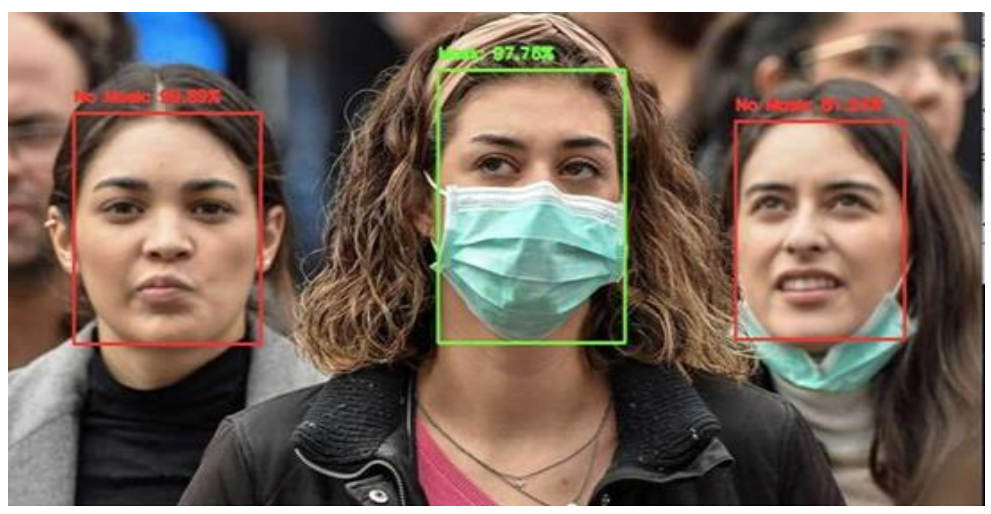

Figure 4. Accuracy of face mask detection

The algorithm worked brilliantly for frontal faces. Figure 5 shows the execution rates for different image resolutions. The graph provides the precision of the face detection rate using OpenCV and compute unified device architecture (CUDA) for various image resolutions. The CUDA platform which is well known for its efficient computation of face detection and recognition tasks was analysed in the proposed system. The blue line indicates the face detection output obtained by the OpenCV function and the red line indicates the detection rate obtained by CUDA based on the Viola-Jones algorithm. The detection was done at a much faster rate and produced outputs precisely than the other approaches and algorithms. The health screening results were obtained within seconds by using the sensors and the output was displayed in the Arduino. Figure 6 shows the health screening results which are the temperature and heart rate of a person.

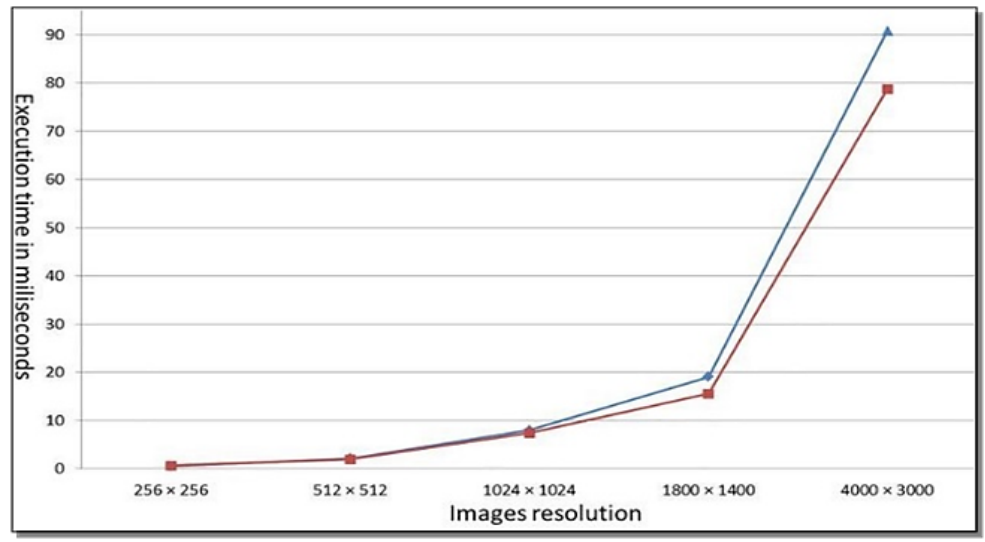

Figure 5. Analysis of Viola-Jones face detection algorithm for various image resolutions

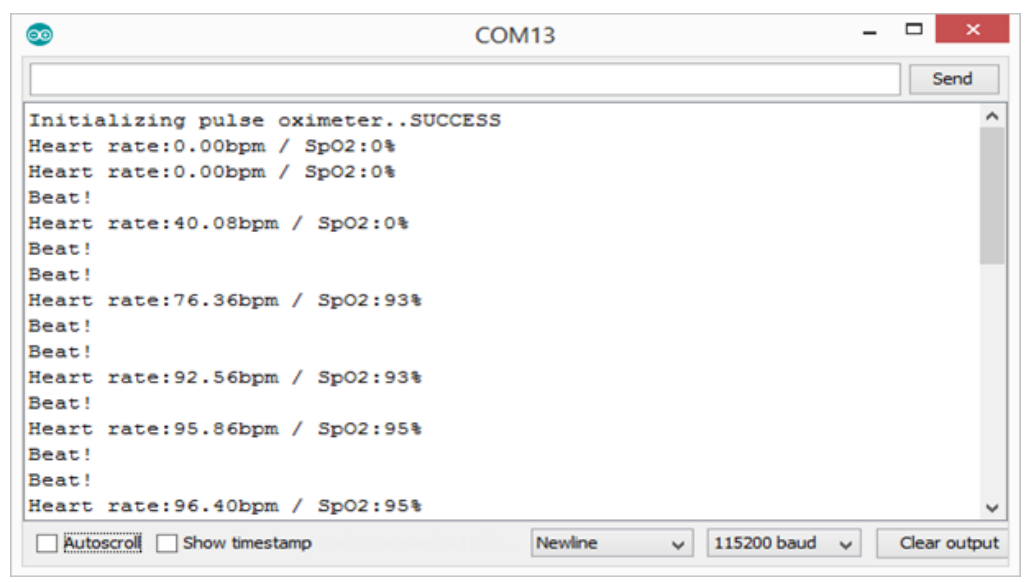

Figure 6. Health screening 


\section{CONCLUSION}

The novel coronavirus is the most fatal and contagious virus affecting humankind. Without the vaccine, it is very tough to battle this deadly disease. Hence this smart solution is proposed to reduce the risk of COVID-19. This device can be placed at any public entrances to ensure whether the people follow the guidelines given by the government without human intervention. The proposed system was able to alert people when face masks were not worn when social distancing was not maintained and alert the authorities related to COVID-19 when any abnormalities found. The project has been implemented with both hardware and software. The hardware containing several sensors were tested with good accuracy. The results obtained were accurate and helped to reduce the risk of the spread of the virus. For future work, the security of the images obtained for facial recognition can be encrypted using the blowfish algorithm and many other image processing techniques can be added for more accurate detection. A wearable band can be designed which can alert people in close proximities not only to a particular public entrance but will also alert the person in any place. The global system for mobile communications (GSM) module can be included for short message service (SMS) alert notifications.

\section{REFERENCES}

[1] R. P. Singh, M. Javaid, A. Haleem, and R. Suman, "Internet of things (IoT) applications to fight against COVID-19 pandemic," Diabetes \& Metabolic Syndrome: Clinical Research \& Reviews, vol. 14, no. 4, pp. 521-524, 2020, doi: 10.1016/j.dsx.2020.04.041.

[2] M. Nasajpour, S. Pouriyeh, R. M. Parizi, M. Dorodchi, M. Valero, and H. R. Arabnia, "Internet of Things for Current COVID-19 and Future Pandemics: An Exploratory Study," Journal of Healthcare Informatics Research, vol. 4, pp. 325-364, 2020, doi: 10.1007/s41666-020-00080-6.

[3] M. M. Islam, S. Mahmud, L. J. Muhammad, M. R. Islam, S. Nooruddin, and S. I. Ayon, "Wearable technology to assist the patients infected with novel coronavirus (COVID-19)," SN Computer Science, vol. 1, no. 6, pp. 1-9, 2020, doi: 10.1007/s42979020-00335-4.

[4] H. Khan, K. K. Kushwah, S. Singh, H. Urkude, M. R. Maurya, and K. K. Sadasivuni, "Smart technologies driven approaches to tackle COVID-19 pandemic: a review," 3 Biotech, vol. 11, no. 2, pp. 1-22, 2021, doi: 10.1007/s13205-020-02581-y.

[5] Y. Yan, A. A. Malik, J. Bayham, E. P. Fenichel, C. Couzens, and S. B. Omer, "Measuring voluntary and policy-induced social distancing behavior during the COVID-19 pandemic," in Proceedings of the National Academy of Sciences, vol. 118, no. 16, 2021, doi: 10.1073/pnas.2008814118.

[6] M. Sethuraman, and M. Senthilkumar, "Preventing Denial-of-service in Next Generation Internet Protocol Mobility," Indonesian Journal of Electrical Engineering and Computer Science (IJEECS), vol. 12, no. 1, pp. 137-146, 2018, doi: 10.11591/ijeecs.v12.i1.pp137-146.

[7] K. Mitchell, "Internet of Things-enabled Smart Devices, Healthcare Body Sensor Networks, and Online Patient Engagement in COVID-19 Prevention, Screening, and Treatment," American Journal of Medical Research, . vol. 8, no. 1, pp. 30-39, 2021;

[8] R. R. Nadikattu, S. M. Mohammad, and D. Whig, "Novel economical social distancing smart device for COVID19," International Journal of Electrical Engineering and Technology; vol. 11, no. 4, 2020.

[9] Hossam A, Magdy A, Fawzy A, and Abd El-Kader SM, "An integrated IoT system to control the spread of COVID-19 in Egypt," Int. Conference on Advanced Intelligent Systems and Informatics, 2020, pp. 336-346, doi: 10.1007/978-3-030-58669-0_31

[10] R. Jaiswal, A. Agarwal, and R. Negi, "Smart solution for reducing the COVID-19 risk using smart city technology," IET Smart Cities, vol. 2, no. 2, pp: 82-88, 2020, doi: 10.1049/iet-smc.2020.0043.

[11] S. Siddiqui, M. Z. Shakir, A. A. Khan, and I. Dey, "Internet of Things (IoT) Enabled Architecture for Social Distancing During Pandemic," Frontiers in Communications and Networks, vol. 2, no. 6, 2021, doi: 10.3389/frcmn.2021.614166.

[12] M. Javaid, A. Haleem, R. Vaishya, S. Bahl, R. Suman, and A. Vaish. "Industry 4.0 technologies and their applications in fighting COVID-19 pandemic,” Diabetes \& Metabolic Syndrome, vol. 14, no. 4, pp. 419-22, 2020, doi: 10.1016/j.dsx.2020.04.032.

[13] R. Stojanović, A. Škraba, and B. Lutovac, “A Headset Like Wearable Device to Track COVID-19 Symptoms," in 2020 9th Mediterranean Conference on Embedded Computing (MECO), 2020, pp. 1-4, doi: 10.1109/MECO49872.2020.9134211.

[14] P. Viola and M. J. Jones, "Robust Real-Time Face Detection," International Journal of Computer Vision, vol. 57, pp. 137-154, 2004, doi: 10.1023/B:VISI.0000013087.49260.fb.

[15] J. Huang, Y. Shang, and H. Chen, "Improved Viola-Jones face detection algorithm based on HoloLens," EURASIP Journal on Image and Video Processing, vol. 41, 2019, doi: 10.1186/s13640-019-0435-6.

[16] H. G. Ziegler, "Privacy-preserving and IoT-capable crowd analysis and detection of flow disturbances for enhancing public safety," 2016 5th Int. Conference on Smart Cities and Green ICT Systems, 2016, pp. 1-8, doi: 10.5220/0005761300550062.

[17] H. G. Hamid and Z. T. Alisa, "A Survey on IoT application layer protocols," Indonesian Journal of Electrical Engineering and Computer Science (IJEECS), vol. 21, no. 3, pp. 1663-1672, 2021, doi: 10.11591/ijeecs.v21.i3.pp1663-1672.

[18] S. Nimmakayala, B. Mummidi, P. Kunda, and S. Kumar, "Modern Health Monitoring System Using IoT," in ICCCE 2020, 2021, pp. 1135-1144, doi: 10.1007/978-981-15-7961-5_105.

[19] M. Botella-Campos, J. Luis Gacía-Navas, A. Rego, S. Sendra, and J. Lloret, "A new system to detect coronavirus social distance violation," International Journal of Electrical and Computer Engineering (IJEECS), vol. 11, no. 6, pp. 5034-5048, 2021, doi: 10.11591/ijece.v11i6.pp5034-5048.

[20] R. Ani, S. Krishna, N. Anju, M. S. Aslam, and O. S. Deepa, "IoT based patient monitoring and diagnostic prediction tool using ensemble classifier," In 2017 International Conference on Advances in Computing, Communications and Informatics (ICACCI), 2017, pp. 1588-1593, doi: 10.1109/ICACCI.2017.8126068.

[21] K. P. Chou et al. "A robust real-time facial alignment system with facial landmarks detection and rectification for multimedia applications," Multimedia Tools and Applications, vol. 80, no. 11, pp. 16635-16657, 2020, doi: 10.1007/s11042-020-09216-7.

[22] R. Tamilkodi and G. R. Nesakumari, "A novel framework for retrieval of image using weighted edge matching algorithm," Multimedia Tools and Applications, vol. 80, no. 13, pp. 1-24, 2021, doi: 10.1007/s11042-020-10452-0.

[23] S. Athira and O.V. M. Ramana, "Face Authentication Using Thermal Imaging," in Computational Vision and Bio Inspired Computing, vol. 28, 2018, doi: 10.1007/978-3-319-71767-8_86. 
[24] C. Almanza, J. M. Baquero, and R. Jiménez-Moreno, "Robotic hex-nut sorting system with deep learning," International Journal of Electrical and Computer Engineering, vol. 11, no. 4, pp. 3575-3583, 2021, doi: 10.11591/ijece.v11i4.pp3575-3583.

[25] M. Saji, M. Sridhar, A. Rajasekaran, R. A. Kumar, A. Suyampulingam, and N. K. Prakash, "IoT-Based Intelligent Healthcare Module," in Advances in Smart System Technologies, vol. 1163, pp.765-774, 2020, doi: 10.1007/978-981-15-5029-4_66.

\section{BIOGRAPHIES OF AUTHORS}
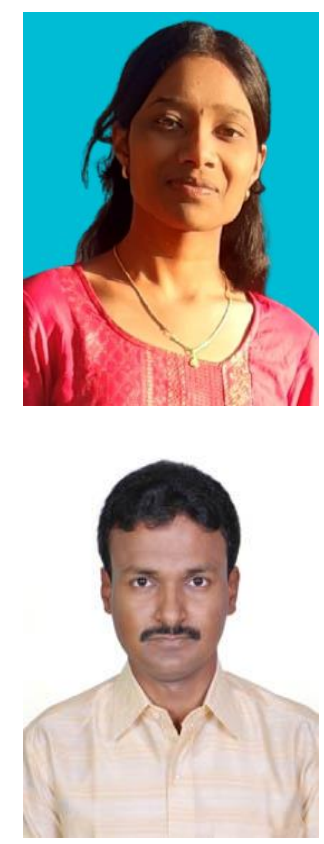

Akshaya Rajeshkumar (iD 8 SC P received the Bachelor's degree in Information technology from Bannari Amman Institute of Technology, Sathyamangalam, Tamil Nadu, India and the Master's degree in Computer Science and Engineering from Amrita School of Engineering, Amrita Vishwa Vidyapeetham, Coimbatore, India. Her research interests include internet of things (IoT), image processing, soft computing, artificial intelligence and machine learning. She can be contacted at email: cb.en.p2cse19002@cb.students.amrita.edu.

Dr. Senthilkumar Mathi (D) SC S P received his Bachelor of Engineering (Computer Science and Engineering) degree from Bharathiar University, India, Master of Engineering (Computer Science and Engineering) degree from Anna University, India, Master of Business Administration (Human Resources Management) degree from Bharathiar University, India and Doctor of Philosophy (under the Faculty of information and communication engineering) degree in Internet Protocol Mobility and Security, from Anna University, India. He is currently Associate Professor in the Department of Computer Science and Engineering, Amrita School of Engineering, Coimbatore Campus, Amrita Vishwa Vidyapeetham, India since 2004. He has around 17 years of teaching and research experience. His areas of interest include IPv4 Mobility and IPv6 Mobility-Next generation internet protocol mobility, machine learning, IoT, mobile computing, cryptography and information security, machine learning and formal languages and automata theory. He has published research papers in reputed journals and conference proceedings, out of which majority of the papers are indexed in SCOPUS, SCI and Web of Science. He has got best paper awards for his publications. He is a member of IETE. He has guided many B.Tech. and M.Tech. student projects or thesis and currently guiding B.Tech. students and M.Tech. and Ph.D. scholars for their project or research or dissertation work. He can be contacted at email: m_senthil@cb.amrita.edu. 